\title{
Current Status of Colorectal Cancer and Its Public Health Burden in Thailand
}

\author{
Kasenee Tiankanon ${ }^{1,2}$, Satimai Aniwan ${ }^{1,2}$ and Rungsun Rerknimitr ${ }^{1,2}$ \\ ${ }^{1}$ Center of Excellence for Innovation and Endoscopy in Gastrointestinal Oncology, Chulalongkorn University, Bangkok, ${ }^{2}$ Division of \\ Gastroenterology, Department of Medicine, Faculty of Medicine, Chulalongkorn University and King Chulalongkorn Memorial Hospital, \\ Thai Red Cross, Bangkok, Thailand
}

Colorectal cancer (CRC) accounts for approximately $10.3 \%$ of new cancer cases in Thailand and is currently the 3rd most prevalent cancer found among the Thai population. Starting in 2017, the Thai government announced the national CRC screening program as a response to this important issue. Among the 70 million people currently residing in Thailand, 14 million require screening, while there are approximately a total of 1,000 endoscopists available to perform colonoscopy. Due to the limited resources and shortage of endoscopists in Thailand, applying a population-based one-step colonoscopy program as a primary screening method is not feasible. To reduce colonoscopy workload, with the help of others, including village health volunteers, institution-based health personnel, reimbursement coders, pathologists, and patients due for CRC screening, a two-step approach of one-time fecal immunochemical test (FIT), which prioritizes and filters out subjects for colonoscopy, is chosen. Moreover, additional adjustments to the optimal FIT cutoff value and the modified Asia-Pacific Colorectal Screening risk score, including body weight, were proposed to stratify the priority of colonoscopy schedule. This article aims to give an overview of the past and current policy developmental strategies and the current status of the Thailand CRC screening program. Clin Endosc 2021;54:499-504

Key Words: Colon cancer; Colon cancer screening; Colonoscopy; Fecal immunochemical test; FIT

\section{INTRODUCTION}

Colorectal cancer $(\mathrm{CRC})$ is one of the most prevalent causes of cancer-related death globally. ${ }^{1}$ In Asian countries, including Thailand, the incidence of CRC has continued to rise during the past few decades. ${ }^{2,3}$ According to the Thai national registry, the burden of CRC is now ranked as the third and fourth most common cancer in males and females, respectively. ${ }^{4}$ Moreover, CRC was the only malignancy with a continuous-

\footnotetext{
Received: September 8, 2020 Revised: October 24, 2020

Accepted: November 13, 2020

Correspondence: Rungsun Rerknimitr

Division of Gastroenterology, Department of Medicine, Faculty of Medicine, Chulalongkorn University and King Chulalongkorn Memorial Hospital, Rama 4 Road, Patumwan, Bangkok 10330, Thailand

Tel: +66-2-256-4265, Fax: +66-2-252-7839, E-mail: ercp@live.com ORCID: https://orcid.org/0000-0001-6866-6886

It is the invited review article.

(c) This is an Open Access article distributed under the terms of the Creative Commons Attribution Non-Commercial License (http://creativecommons.org/ licenses/by-nc/3.0) which permits unrestricted non-commercial use, distribution, and reproduction in any medium, provided the original work is properly cited.
}

ly growing incidence in both sexes (average annual percent changes in males $=4.1 \%$ and females $=3.3 \%) .{ }^{5}$ In 2017 , the age-standardized incidence rates for colon cancer were 14.1 per 100,000 person-years ( $55 \%$ increase) in men and 10 per 100,000 person-years (19\% increase) in women. ${ }^{5}$ In 2018 , 17,534 patients with newly diagnosed CRC were reported, accounting for $10.3 \%$ of all new cancer cases in Thailand that year. ${ }^{4}$

Even though CRC is a major public health problem and has an enormous burden on society, it is a good candidate for mass cancer screening programs for multiple reasons. Most CRCs begin with small colonic adenomatous polyps that then develop malignant properties over time; therefore, early detection and removal of these colonic adenomas can prevent CRC. ${ }^{6}$ Moreover, even if CRC has already developed, it can still be cured if it is detected and removed at an early stage. In response to the threat of CRC, the Thai government announced an official national CRC screening and treatment program in October 2017. However, in developing countries with limited resources, such as Thailand, the establishment of 
a practical CRC screening program that can be integrated into pre-existing national public health services is still a challenge.

This article aims to provide an overview of the past and current status of CRC screening programs in Thailand and policy strategies to address the difficulties and challenges of these programs.

\section{COLORECTAL CANCER SCREENING PROTOCOLS}

Because of the importance of CRC, the Thai Ministry of Public Health and the National Cancer Institute of Thailand have aimed to use primary and secondary prevention to control the disease. Several tests and procedures have been proposed for CRC screening, but only two protocols are currently accepted.

One CRC screening method is primary colonoscopy, which is considered the most accurate test for early cancer detection and prevention, with many strong evidences. ${ }^{7-10}$ The advantage of endoscopic screening is that it is a one-step approach for the detection and removal of polyps, and the benefits last up to ten years. However, colonoscopies are invasive and they require considerable investments in expensive resources, including colonoscopes, colonoscopy accessories, and endoscopy units. In addition, performing a colonoscopy also requires welltrained endoscopists, endoscopic nurses, and anesthesiological personnel, on some occasions.

It is noteworthy that endoscopy units and colonoscopists are quite scarce in Thailand. The number of board-certified endoscopists in the country is currently lower than 1,000 . Meanwhile, among the Thai population of 70 million, the estimated number of cases for CRC screening is 14 million. ${ }^{11}$ Evi- dently, demand outnumbers the supply. Even if the strategy of performing a single colonoscopy in the lifetime of each person who has a first-degree relative with CRC was to be chosen, the additional workload would rise to almost 10 more colonoscopies/week/colonoscopist. Limited by financial constraints and a shortage of endoscopists, applying colonoscopy as a primary screening method for population-based screening programs is not feasible. ${ }^{12}$

Another method for CRC screening is the fecal immunochemical test (FIT). FIT is a monoclonal or polyclonal antibody against human hemoglobin used for direct measurements of human blood in stool. FIT has a sensitivity of $34 \%-57 \%$ for advanced neoplasia and a specificity (91\%-95\%) comparable to that of a colonoscopy. ${ }^{13}$ Due to its non-invasive nature, affordability, high sensitivity, simplicity, and the fact that it does not require any dietary restrictions, FIT was proposed as a suitable and better alternative to colonoscopies. ${ }^{14}$ As a more practical option and to reduce colonoscopy workload, a two-step approach in which a one-time FIT test is used to prioritize subjects for colonoscopy is chosen.

FIT-based screening is a process that requires participation, compliance, and adherence by all sectors involved in the process. Fortunately, Thailand has well-developed public health services with extensive primary care networks that are well integrated with districts, provincial hospitals, and tertiary care centers. In a small social unit like villages, village health volunteers recruited from local communities play an important role in enhancing community engagement. Moreover, at the nationwide level, the entire Thai population is covered by universal health coverage, a comprehensive healthcare package covering most of healthcare expenses, including diagnostic, preventive, treatment, and follow-up costs. ${ }^{15}$ Despite the lack of endoscopists, the country receives help from others, includ-

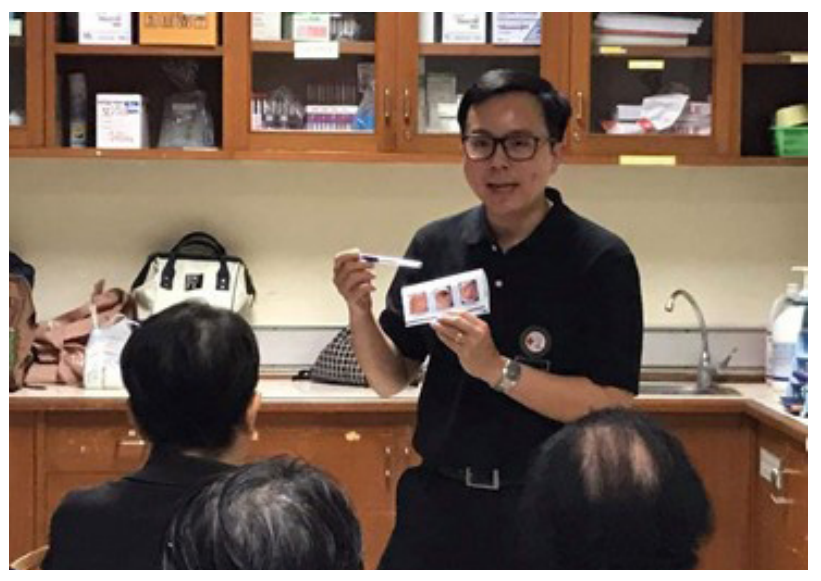

Fig. 1. Bowel preparation quality introduction by medical personnel.

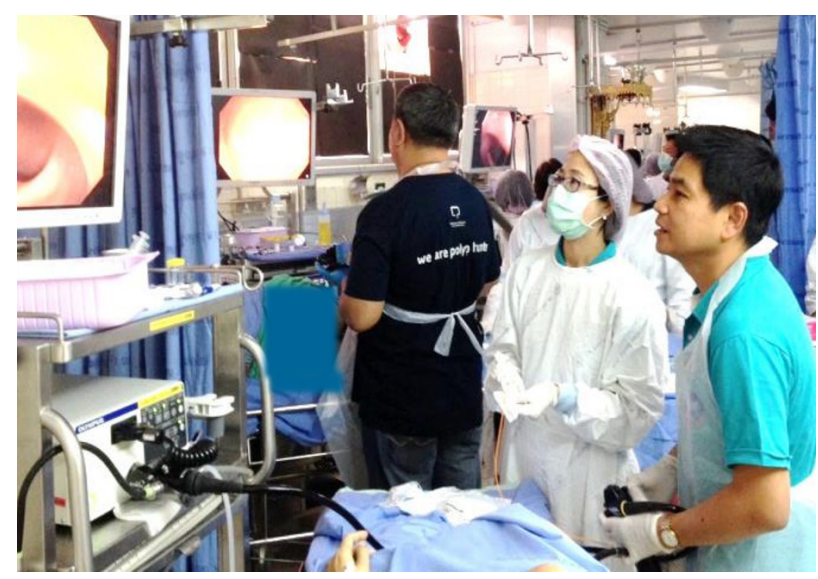

Fig. 2. Mass screening colonoscopy with 100 procedures per day. 
ing village health volunteers, institution-based health personnel, reimbursement coders, pathologists, and patients due for CRC screening (Figs. 1 and 2).

In 2014, the pilot CRC screening project was launched in one of the northern provinces of Thailand, Lampang. ${ }^{16}$ The initial protocol used a $200 \mathrm{mg} / \mathrm{mL}$ cutoff, one-time quantitative FIT test. A total of 127,301 test kits were sent to all asymptomatic and apparently healthy subjects with no history of CRC aged 50-65 years. A total of 80,012 specimens (63\%) from 36,601 (57.8\%) men and 43,411 (67.8\%) women were returned. Among the returned specimens, 876 (1.1\%) were FIT positive. Subsequently, 72\% (627 patients) of FIT positive patients eventually underwent colonoscopy, revealing 23 cancers (3.7\%), 75 (12\%) advanced adenomas, and 187 (20\%) adenomas. The successful implementation of the pilot CRC screening with satisfactory process measures indicates the feasibility of scaling-up organized CRC screening through existing health services in Thailand. ${ }^{16}$

In 2014, a national survey reported that there were 10 million Thai individuals aged $>50$ years of age who were eligible for CRC screening. Based on the data from a pilot study, which reported a positive FIT rate of approximately $12 \%$, when applying this strategy among the Thai population in need of screening, the number of colonoscopies would decrease to 1.2 million per year. ${ }^{17}$ Although FIT alone can reduce the workload by up to 8 -fold, it was estimated that, if the entire targeted population of Thailand was enrolled in the FIT-based CRC screening program, and with a participation rate of approximately $70 \%$, such as in the pilot study, it would still take 8 to 12 years to screen all of the target populations. ${ }^{18}$ This is because in a country with limited resources, such as Thailand, the healthcare system can handle less than 200,000 colonoscopies per year. Therefore, further sub-stratification for prioritizing subjects is mandated.

To sub-stratify subjects, the Asia-Pacific Colorectal Screening (APCS) score was proposed as a tool for selecting high-risk participants who might have advanced neoplasia. According to the APCS score, ${ }^{12}$ four traditional factors, including gender, age, smoking, and family history of colon cancer were quoted as the risk factors for the development of CRC. Patients who were classified as having high or moderate risk demonstrated that the relative risk of finding advanced neoplasia was 2.6-fold to 4.3 -fold higher than in low-risk participants.

Therefore, to select and prioritize subjects at high risk of advanced neoplasia for colonoscopy, our research group studied the benefit of a combination of FIT and clinical risk scores. ${ }^{17}$ This study aimed to determine the prevalence of colorectal neoplasia in 4 different groups by using a FIT hemoglobin detection cut off of $50 \mathrm{ng} / \mathrm{mL}$ (positive FIT vs. negative FIT) and APCS scores (high-risk vs. average-risk). We stratified 957 asymptomatic subjects who presented for CRC screening into 4 groups. The first group contained patients with high risk and positive FIT ( $n=84 ; 8.9 \%)$; the second group contained patients with high risk but negative FIT $(n=173 ; 18.2 \%)$; the third group contained patients with an average risk but positive FIT ( $n=192 ; 20.3 \%$ ); and the last group contained patients with an average risk and negative FIT $(n=499 ; 52.6 \%)$. All patients underwent a colonoscopy, which was performed by experienced endoscopists who had performed more than 1,000 colonoscopies, with an acceptable adenoma detection rate (adenoma detection rate $>20 \%$ ). We found that the prevalence of advanced adenoma in groups $1,2,3$, and 4 were $36.9 \%, 11.6 \%$, $12.0 \%$, and $6.4 \%$, respectively. ${ }^{17}$ Subjects in group 1 had a significantly higher detection rate of advanced neoplasia compared to the other 3 groups (6.15-fold, 95\% confidence interval, 3.72-10.17). In this study, no cancer was found in group 4. Seven cancers were discovered in the study; 4 were found in the group 1, 1 in group 2, and 2 in group 3. Evidently, the result demonstrated the synergistic effect of combining FIT with the clinical risk score. This result further suggests that those with positive FIT results and high-risk scores should be prioritized for colonoscopy, while those with either high-risk scores alone or positive FIT results alone can wait to undergo a colonoscopy. The latter 2 subgroups shared a similar rate (12\%) of advanced neoplasia. In addition, if colonoscopy resources allow, subjects with negative FIT results and an average-risk score could be the last group to undergo colonoscopy. ${ }^{17}$

After this successful pilot study, an official nationwide Thailand CRC screening program was launched in October 2017 which used a one-time FIT with an initial cutoff level of 100 $\mathrm{ng} / \mathrm{mL}$. However, preliminary results suggest that the optimal FIT cutoff level for CRC screening in Thailand must be adjusted according to colonoscopy resources and the target population size. ${ }^{19-21}$ Different cut-off hemoglobin levels (range, 10-75 $\mathrm{ng} / \mathrm{mL}$ ) provide different positive rates of FIT (range, $4.5 \%$ to $46.4 \%$ ) which are then used as the basis for colonoscopy referral. ${ }^{17,22}$ Hypothetically, a higher cutoff value would result in a lower workload. However, the tradeoff for this adjustment is the decrease in the sensitivity and the chance of missing advanced adenoma and cancer. To decrease the colonoscopy workload and enhance effective FIT-based CRC screening, optimal cutoff levels should be individualized based on risk factors.

To determine the optimal cutoff level for our country, we conducted a multi-center study in 6 hospitals across Thailand in 2017. ${ }^{23}$ The study assessed test performance in detecting advanced adenoma and CRC with FIT cutoff values of 25, 50, 100, 150, and $200 \mathrm{ng} / \mathrm{mL}$. Among 1,479 patients, adenoma, 
advanced neoplasia, and CRC were present in 547 (37\%), 137 (9.3\%), and $14(0.9 \%)$ participants, respectively. The study revealed that cut-offs from $25 \mathrm{ng} / \mathrm{mL}$ to $200 \mathrm{ng} / \mathrm{mL}$ had both relatively high sensitivity (range, 64.3\%-78.6\%) and specificity (range, 82.3\%-95.6\%) for CRC and fair sensitivity (range, $16.8 \%-42.3 \%$ ) with high specificity (range, $84.2 \%-96.3 \%$ ) for advanced neoplasia. For CRC detection, cutoffs at $150 \mathrm{ng} /$ $\mathrm{mL}$ and $200 \mathrm{ng} / \mathrm{mL}$ yielded similar positive predictive values ( $12.5 \%$ vs. $12.3 \%)$, negative predictive values $(99.8 \%$ vs. 99.6\%), and numbers needed for a colonoscopy to find one CRC (8.0 vs. 8.1). When the CRC miss rate was taken into account, decreasing the cut-off from $150 \mathrm{ng} / \mathrm{mL}$ to $25 \mathrm{ng} / \mathrm{mL}$ did not increase the CRC detection rate (CRC miss rates $[n=3]$ $21 \%$ ), whereas increasing the cut-off from $150 \mathrm{ng} / \mathrm{mL}$ to 200 $\mathrm{ng} / \mathrm{mL}$ resulted in an increased CRC miss rate to $35 \%(n=5)$. Based on these results, we propose that the optimal FIT cutoff for Thailand should be $150 \mathrm{ng} / \mathrm{mL}$ because it offers both high positive and negative predictive values for advanced neoplasia detection and reduced colonoscopy workload without increasing the rate of missed $\mathrm{CRC}$. $^{23}$

After the recommendation of increasing the cut off value, some experts have raised concerns and suggested that the FIT cutoff value for high risk patients should be lowered in order to avoid missing significant lesions. ${ }^{23}$ To answer this question, another multi-center prospective study evaluated the diagnostic performance of the FIT at different cutoffs in high-risk subjects, as defined by the APCS scoring system, compared to average-risk subjects. ${ }^{24} \mathrm{~A}$ total of 1,713 patients were recruited for the study. A total of 1,222 (71.3\%) subjects were classified as average-risk and 491 (28.7\%) subjects were high-risk. At the cutoff values of $25,50,100,150$, and $200 \mathrm{ng} / \mathrm{mL}$, the average sensitivities for the detection of advanced neoplasia decreased from $42 \%$ to $32 \%, 23 \%, 18 \%$, and $17 \%$, respectively. At the cutoff values of $25 \mathrm{ng} / \mathrm{mL}$ and $150 \mathrm{ng} / \mathrm{mL}$, patients in the high-risk group yielded significantly higher sensitivities than those in the average-risk group (cutoffs at $25 \mathrm{ng} / \mathrm{mL}=$ $52.3 \%$ vs. $34.4 \%, p=0.03$; cutoff at $150 \mathrm{ng} / \mathrm{mL}=32.3 \%$ vs. $17.8 \% ; p=0.04) .{ }^{24}$ At this cutoff, high-risk patients still yielded comparable specificity for advanced neoplasia (92\%) and high negative predictive values to reject the risk of colon cancers $(100 \%) .{ }^{24}$ At a cut-off of $25 \mathrm{ng} / \mathrm{mL}$, the number of needed colonoscopies to find one advanced neoplasia for the highrisk and average-risk groups was 2.8 and 6.1, respectively. As a result, our study suggests that using the $25 \mathrm{ng} / \mathrm{mL}$ cutoff for high-risk patients and the $150 \mathrm{ng} / \mathrm{mL}$ cutoff for average-risk patients could maintain the sensitivity for CRC $(80 \%)$ (Table $1){ }^{23}$ Recently, we initiated a large population-based study evaluating the two-step CRC screening approach with a combination of different FIT cutoffs and clinical risk stratification. This study is ongoing.

Evidence from previous studies, including Western and Asian studies, demonstrated the association between the risk of colorectal adenoma development and obesity. ${ }^{25}$ Even though the prevalence of obesity in Thailand is not as high as in Western countries, it has been increasing. According to a 2009 national survey, the prevalence of obesity with body mass index (BMI) $\geq 25 \mathrm{~kg} / \mathrm{m}^{2}$ in adults increased dramatically from $18.2 \%$ in 1991 to $24.1 \%$ in 1997 and $28.1 \%$ in 2004 . For those with BMI $\geq 30 \mathrm{~kg} / \mathrm{m}^{2}$, the prevalence increased from $3.5 \%$ to $5.8 \%$ to $6.9 \%$ in the corresponding years. ${ }^{26}$ Therefore, Thailand is facing an increasing prevalence of overweight population. In addition to traditional risk factors for CRC, being overweight was proposed as another important factor contributing to the development of CRC. ${ }^{27}$

As a result, our research group conducted a prospective cross-sectional study in 2019 to evaluate the efficacy of colorectal neoplasia detection using the modified APCS score. The modified APCS score includes a combination of traditional risk factors of from APCS score plus the overweight risk factor. $^{28} \mathrm{~A}$ total of 338 asymptomatic subjects, aged $50-75$ years of age, who attended the CRC screening clinic were included in the study. Subjects were categorized into an average-risk group and a high-risk group according to the original APCS score. BMI cut-off at $23 \mathrm{~kg} / \mathrm{m}^{2}$ for the Asian population was used to define overweight subjects according to $\mathrm{WHO}$ expert consultation criteria. ${ }^{29}$ Based on this criterion, 192 subjects (57 \%) were

Table 1. Colorectal Cancer Screening Protocol in Thailand

\begin{tabular}{|c|c|}
\hline Target population $>50$ years & 14 million \\
\hline Persons involved & $\begin{array}{l}\text { Village health volunteers, institution-based health personnel, } \\
\text { reimbursement coders, pathologists, patients due for screening }\end{array}$ \\
\hline The Asia-Pacific Colorectal Screening score & Gender, age, smoking, and family history of colon cancer \\
\hline Fecal immunochemical test cutoff vale for average- risk group & $150 \mathrm{ng} / \mathrm{mL}$ \\
\hline Fecal immunochemical test cutoff vale for high-risk group & $25 \mathrm{ng} / \mathrm{mL}$ \\
\hline Overweight (body mass index $>23 \mathrm{~kg} / \mathrm{m}^{2}$ ) & Increase in colorectal cancer risk \\
\hline
\end{tabular}


defined as overweight. The detection rates of adenoma and advanced adenoma in overweight subjects were significantly higher than those in subjects with normal weight (for adenoma $44 \%$ vs. $24 \%, p<0.01$; for advanced adenoma, $12 \%$ vs. $2 \%$, $p<0.01$, respectively). ${ }^{28}$ Using the combination of the original APCS score and the overweight risk factor, adenoma detection rates significantly varied among the 4 groups: $64 \%$ in the high-risk with overweight group, $40 \%$ in the average-risk with overweight group, $32 \%$ in the high-risk with normal weight group, and $21 \%$ in the average-risk and normal weight group $(p<0.01){ }^{28}$ In other words, the result demonstrated the synergistic effect of combining APCS score and being overweight for the prediction of colorectal neoplasia detection. However, before being integrated into routine practice, the modified APCS score requires further validation by comparing it with the standard APCS score.

Lastly, FIT-based CRC screening in Thailand will only be effective when a high proportion of subjects with a positive result uptake further diagnostic colonoscopy. In addition, it is vital for the national database registry to evaluate longterm outcomes, including CRC incidence and mortality. Our nationwide CRC screening program can still be further improved through various methods such as training more endoscopists, investing in endoscopy units, and providing better quality measurement and reimbursement systems. For the ultimate goal of reducing the incidence of CRC and CRC-related mortality in Thailand in the future, ongoing and future research is needed to help develop better policies for primary and secondary prevention.

\section{CONCLUSIONS}

CRC is one of the most important healthcare burdens in Thailand. Due to the fact that Thailand is a country with limited-resources, the two-step approach led by FIT has been chosen. However, additional adjustments to the FIT cutoff value and clinical risk stratification may be used to stratify the priority of the colonoscopy schedule. However, the benefits of this approach in Thailand will not be seen until a few more decades.

\section{Conflicts of Interest}

Rungsun Rerknimitr has been an Editorial Board member of Clinical Endoscopy; however, he was not involved in the peer reviewer selection, evaluation, or decision process of this article. Otherwise, no other potential conflicts of interest relevant to this article was reported.

$$
\begin{gathered}
\text { Funding } \\
\text { None. }
\end{gathered}
$$

\section{Author Contributions}

Conceptualization: Satimai Aniwan, Rungsun Rerknimitr

Data curation: Kasenee Tiankanon

Formal analysis: SA

Funding acquisition: RR

Project administration: SA, RR

Writing-original draft: KT, SA

Writing-review\&editing: KT, SA, RR

\section{ORCID}

Kasenee Tiankanon:

Rungsun Rerknimitr:
Satimai Aniwan: https://orcid.org/0000-0001-8550-8142 https://orcid.org/0000-0002-2730-2450 https://orcid.org/0000-0001-6866-6886

\section{REFERENCES}

1. Ferlay J, Shin HR, Bray F, Forman D, Mathers C, Parkin DM. Estimates of worldwide burden of cancer in 2008: GLOBOCAN 2008. Int J Cancer 2010;127:2893-2917.

2. Khuhaprema T, Srivatanakul P. Colon and rectum cancer in Thailand: an overview. Jpn J Clin Oncol 2008;38:237-243.

3. Phiphatpatthamaamphan K, Vilaichone R. Colorectal cancer in the central region of Thailand. Asian Pac J Cancer Prev 2016;17:3647-3650.

4. Bray F, Ferlay J, Soerjomataram I, Siegel RL, Torre LA, Jemal A. Global cancer statistics 2018: GLOBOCAN estimates of incidence and mortality worldwide for 36 cancers in 185 countries. CA Cancer J Clin 2018;68:394-424.

5. Virani S, Bilheem S, Chansaard W, et al. National and subnational population-based incidence of cancer in Thailand: assessing cancers with the highest burdens. Cancers (Basel) 2017;9:108.

6. Levin B, Lieberman DA, McFarland B, et al. Screening and surveillance for the early detection of colorectal cancer and adenomatous polyps, 2008: a joint guideline from the American Cancer Society, the US Multi-Society Task Force on Colorectal Cancer, and the American College of Radiology. CA Cancer J Clin 2008;58:130-160.

7. Winawer SJ, Zauber AG, O'Brien MJ, et al. Randomized comparison of surveillance intervals after colonoscopic removal of newly diagnosed adenomatous polyps. The national polyp study workgroup. N Engl J Med 1993;328:901-906.

8. Winawer SJ, Zauber AG, Ho MN, et al. Prevention of colorectal cancer by colonoscopic polypectomy. The national polyp study workgroup. $\mathrm{N}$ Engl J Med 1993;329:1977-1981.

9. Kavanagh AM, Giovannucci EL, Fuchs CS, Colditz GA. Screening endoscopy and risk of colorectal cancer in United States men. Cancer Causes Control 1998;9:455-462.

10. Levin B, Lieberman DA, McFarland B, et al. Screening and surveillance for the early detection of colorectal cancer and adenomatous polyps, 2008: a joint guideline from the American Cancer Society, the US Multi-Society Task Force on Colorectal Cancer, and the American College of Radiology. Gastroenterology 2008;134:1570-1595.

11. Khuhaprema T, Attasara P, Sriplung H, Wiangnon S, Sangrajrang S.Cancer in Thailand: volume VII (2007-2009) [Internet]. Bangkok: National Cancer Institute; c2013 [cited 2020 Aug 10]. Available from: http://www. nci.go.th/th/File_download/Nci\%20Cancer\%20Registry/Cancer\%20 in\%20thailand_VII.pdf.

12. Yeoh KG, Ho KY, Chiu HM, et al. The Asia-Pacific colorectal screening score: a validated tool that stratifies risk for colorectal advanced neoplasia in asymptomatic Asian subjects. Gut 2011;60:1236-1241.

13. Parra-Blanco A, Gimeno-García AZ, Quintero E, et al. Diagnostic accuracy of immunochemical versus guaiac faecal occult blood tests for colorectal cancer screening. J Gastroenterol 2010;45:703-712. 
14. Robertson DJ, Lee JK, Boland CR, et al. Recommendations on fecal immunochemical testing to screen for colorectal neoplasia: a consensus statement by the US Multi-Society Task Force on Colorectal Cancer. Gastroenterology 2017;152:1217-1237.e3.

15. Hanvoravongchai P. Health financing reform in Thailand: toward universal coverage under fiscal constraints [Internet]. Washington DC: World Banks; c2013 [cited 2021 Feb 18]. Available from: https://openknowledge.worldbank.org/handle/10986/13297.

16. Khuhaprema T, Sangrajrang S, Lalitwongsa S, et al. Organised colorectal cancer screening in Lampang Province, Thailand: preliminary results from a pilot implementation programme. BMJ Open 2014;4:e003671.

17. Aniwan S, Rerknimitr R, Kongkam P, et al. A combination of clinical risk stratification and fecal immunochemical test results to prioritize colonoscopy screening in asymptomatic participants. Gastrointest Endosc 2015;81:719-727.

18. Saengow U, Chongsuwiwatvong V, Geater A, Birch S. Preferences and acceptance of colorectal cancer screening in Thailand. Asian Pac J Cancer Prev 2015;16:2269-2276.

19. Alvarez-Urturi C, Andreu M, Hernandez C, et al. Impact of age-and gender-specific cut-off values for the fecal immunochemical test for hemoglobin in colorectal cancer screening. Dig Liver Dis 2016;48:542-551.

20. Auge JM, Pellise M, Escudero JM, et al. Risk stratification for advanced colorectal neoplasia according to fecal hemoglobin concentration in a colorectal cancer screening program. Gastroenterology 2014;147:628636.e1.

21. Kapidzic A, van der Meulen MP, Hol L, et al. Gender differences in fecal immunochemical test performance for early detection of colorectal neo- plasia. Clin Gastroenterol Hepatol 2015;13:1464-1471.e4.

22. Hundt S, Haug U, Brenner H. Comparative evaluation of immunochemical fecal occult blood tests for colorectal adenoma detection. Ann Intern Med 2009;150:162-169.

23. Aniwan S, Ratanachu Ek T, Pongprasobchai S, et al. The optimal cut-off level of the fecal immunochemical test for colorectal cancer screening in a country with limited colonoscopy resources: a multi-center study from Thailand. Asian Pac J Cancer Prev 2017;18:405-412.

24. Aniwan S, Ratanachu-Ek T, Pongprasobchai S, et al. Impact of fecal $\mathrm{Hb}$ levels on advanced neoplasia detection and the diagnostic miss rate for colorectal cancer screening in high-risk vs. average-risk subjects: a multi-center study. Clin Transl Gastroenterol 2017;8:e113.

25. Okabayashi K, Ashrafian H, Hasegawa H, et al. Body mass index category as a risk factor for colorectal adenomas: a systematic review and meta-analysis. Am J Gastroenterol 2012;107:1175-1185; quiz 1186.

26. Aekplakorn W, Mo-Suwan L. Prevalence of obesity in Thailand. Obes Rev 2009;10:589-592.

27. Harriss DJ, Atkinson G, George K, et al. Lifestyle factors and colorectal cancer risk (1): systematic review and meta-analysis of associations with body mass index. Colorectal Dis 2009;11:547-563.

28. Jangsirikul S, Promratpan W, Aniwan S, et al. Overweight as an additional risk factor for colorectal neoplasia in lean population. Asian Pac J Cancer Prev 2019;20:105-111.

29. WHO Expert Consultation. Appropriate body-mass index for Asian populations and its implications for policy and intervention strategies. Lancet 2004;363:157-163. 